\title{
AN EXPLICIT SUMMATION FORMULA AND ITS APPLICATION
}

\section{LEON BERNSTEIN}

1. Summary of results. It is the purpose of this paper to state and prove an explicit formula which has a wide range of application to some significant problems in Number Theory, Algebra, Probability and Analysis. In various domains of these disciplines many problems, otherwise solved, have been anticipating such an explicit solution. ${ }^{1}$ The following results are obtained:

(i) The only positive real root of the irreducible polynomial

$$
P_{n}(x)=x^{n}+x^{n-1}+\cdots+x-1 \quad(n>1)
$$

is stated explicitly.

(ii) Let be

$$
P_{n}\left(x_{i}\right)=0 \quad(i=1, \cdots, n),
$$

then the partition polynomials of degree $m>0$ are defined by the symmetric functions

$$
\begin{gathered}
g_{m}\left(x_{1}, x_{2}, \cdots, x_{n}\right)=\sum\left(x_{1}^{m_{1}} x_{2}^{m_{2}} \cdots x_{n}^{m_{n}}\right)^{-1} \\
\sum \text { extended over all nonnegative } m_{i} \text { for which } \\
m_{1}+2 m_{2}+\cdots+n m_{n}=m
\end{gathered}
$$

then the numeric values of $g_{m}$ are stated explicitly for any $m$.

(iii) The generalized Fibonacci numbers of order $n>1$ are defined by initial values and recurrency formulas as follows:

$$
\begin{aligned}
& F_{1}^{(n)}=F_{2}^{(n)}=\cdots=F_{n-1}^{(n)}=0, \quad F_{n}^{(n)}=1, \\
& F_{n+k}^{(n)}=\sum_{j=0}^{n-1} F_{k+j}^{(n)} \quad(k=1,2, \cdots),
\end{aligned}
$$

the values of $F_{n+k}^{(n)}$ are stated explicitly for any $k>0$ as functions of $n$ and $k$ (including the original Fibonacci numbers for $n=2$ ).

(iv) The probability that a fragile stick of $s n+k(s=0,1, \cdots$; $k=1, \cdots, n$ ) units length, breakable in to pieces each consisting of an integral number of units not exceeding $n$, would break into $y_{1}$

Received by the editors December 5, 1968.

1 The author wishes to express his gratitude to Professor Helmut Hasse for valuable assistance in proving this formula. 
pieces of one unit length each, into $y_{2}$ pieces of two units length each, $\cdots$, into $y_{n}$ pieces of $n$ units length each, is stated as an explicit function of the parameters $y_{1}, \cdots, y_{n}, s, n, k$.

2. Some previous results of the author. We shall recall the Generalized Algorithm of Jacobi-Perron [4], [5] which is defined as follows: Let $E_{n-1}$ be the real Euclidean vector space of dimension $n-1>0$. Let

$$
a^{(v)}=\left(a_{1}^{(v)}, \cdots, a_{n-1}^{(v)}\right), \quad b^{(v)}=\left(b_{1}^{(v)}, \cdots, b_{n-1}^{(v)}\right)
$$

$(v=0,1, \cdots)$ be two sequences of vectors with $a^{(0)}$ a fixed vector in $E_{n-1}$, while the vectors $a^{(v+1)}$ are derived from $a^{(0)}$ and $b^{(v)}$ in the following way: let $T$ be a transformation mapping $E_{n-1}$ into $E_{n-1}$ by the formula

$$
\begin{array}{r}
T a^{(v)}=\left(a_{1}^{(v)}-b_{1}^{(v)}\right)^{-1}\left(a_{2}^{(v)}-b_{2}^{(v)}, \cdots, a_{n-1}^{(v)}-b_{n-1}^{(v)}, 1\right)=a^{(v+1)} \\
\left(a_{1}^{(v)} \neq b_{1}^{(v)}, v=0,1, \cdots\right) .
\end{array}
$$

The successive transformation $T^{k} a^{(0)}=T\left(T^{k-1} a^{(0)}\right)(k=2,3, \cdots)$ is called the Generalized Algorithm of Jacobi-Perron. We shall write $T^{k}$ for $T^{k} a^{(0)}$ where no ambiguity is involved. The algorithm $T^{k}$ is called periodic, if there exist nonnegative integers $Q, M$

$$
Q \geqq 0, \quad M \geqq 1 \quad \text { with } \min Q=q, \quad \min M=m
$$

such that

$$
T^{v+m}=T^{v} \quad(v=q, q+1, \cdots) .
$$

The vectors $b^{(v)}(v=0,1, \cdots, q-1)$ are called the primitive preperiod of the algorithm $T^{k}$, and the $m$ vectors $b^{(q+v)}(v=0,1, \cdots$, $m-1)$ its primitive period. If $q=0, T^{k}$ is called purely periodic. The transformation matrix of $T^{k}$ is generated by the numbers $A_{i}^{(v)}$ which are defined by the identity matrix and recursion formula

$$
\begin{gathered}
A_{i}^{(i)}=1, \quad A_{i}^{(v)}=0 \quad(i \neq v ; \quad i, v=0,1, \cdots, n-1), \\
A_{i}^{(v+n)}=\sum_{j=0}^{n-1} b_{j}^{(v)} A_{i}{ }^{(v+j)} \\
\quad\left(b_{0}{ }^{(v)}=1 ; \quad i=0, \cdots, n-1 ; \quad v=0,1, \cdots\right) .
\end{gathered}
$$

In [1] the author has proved the following theorems:

THEOREM $[1,1]$. Let w be a root of the monic polynomial with rational integral coefficients 


$$
F_{n}(x)=x^{n}+k_{1} x^{n-1}+\cdots+k_{n-1} x-d \quad(d \neq 0),
$$

let the vector $a^{(0)} \in E_{n-1}$ have the components

$$
a_{s}^{(0)}=a_{s}^{(0)}(w)=\sum_{i=0}^{s} k_{i} w^{s-i} \quad\left(k_{0}=1 ; s=1, \cdots, n-1\right),
$$

let the vectors $b^{(v)}$ be derived from the vectors $a^{(v)}$ by the formula

$$
b_{s}^{(v)}=a_{s}^{(v)}(0) \quad(s=1, \cdots, n-1) .
$$

Then the algorithm $T^{k} a^{(0)}$ is purely periodic; the length of the primitive period equals

$$
m=n \quad \text { if } d \neq 1, \quad=1 \quad \text { if } d=1 .
$$

For $d \neq 1$, the primitive period vectors have the form

(i) $b^{(0)}=\left(k_{1}, k_{2}, \cdots, k_{n-1}\right)$,

$$
\text { (ii) } \begin{array}{r}
b^{(s)}=\left(k_{1}, \cdots, k_{n-s-1}, d^{-1} k_{n-s}, d^{-1} k_{n-s+1}, \cdots, d^{-1} k_{n-1}\right) \\
(s=1, \cdots, n-1) .
\end{array}
$$

For $d=1$, the primitive period vector has the form (2.10) (i).

ThEOREM $[1,2]$. Let the coefficients of the polynomial $F_{n}(x)$ in (2.6) be such that

$$
k_{i} \geqq 0 \quad(i=1, \cdots, n-2), \quad k_{n-1}, \quad d \geqq 1,
$$

and let w be a positive root of $F_{n}(x)$ such that $0<w<1$.

Then the algorithm $T^{k} a^{(0)}$ is convergent in the sense that

$$
w=\lim _{v \rightarrow \infty}\left(u A_{0}^{(v-1)} / A_{0}^{(v)}\right), \quad \begin{aligned}
& u=d, \quad \text { if } v \equiv 0 \quad(\bmod n), \\
& u=1 \text { otherwise, }
\end{aligned}
$$

where the numbers $A_{0}^{(0)}$ are constructed from the elements of the primitive period (2.10) by means of the recursion formulas (2.5).

Let now the coefficients of $F_{n}(x)$ from (2.6) have the form $k_{i}, d=1$ $(i=1, \cdots, n-1)$ and let $w$ be the (only) real positive root of $F_{n}(x)$. Then, by Theorem $[1,1]$, the algorithm $T^{k} a^{(0)}$, where

$$
a^{(0)}=\left(w+1, w^{2}+w+1, \cdots, w^{n-1}+w^{n-2}+\cdots+w+1\right)
$$

is purely periodic and the primitive period whose length is now one has the form

$$
b^{(v)}=(1,1, \cdots, 1) \quad(v=0,1, \cdots) .
$$


Let $A_{01}^{(0)}$ be the $A_{0}^{(0)}$ constructed from the elements of (2.13) by means of the recursion formula (2.5). Then, as has been proved by the author in [2], for the Generalized Fibonacci Numbers (1.4) the relation holds

$$
F_{m}^{(n)}=A_{01}^{(m)} \quad(m=1,2, \cdots ; n=2,3, \cdots) .
$$

In [3] the author has proved

THEOREM $[2,1]$. The probability that a fragile stick of $s n+k(s, k$ as in (iv)) units length, breakable into pieces, each consisting of an integral number of units not exceeding $n$, would break into $x_{1}$ pieces of one unit length, into $x_{2}$ pieces of two units length, $\cdots, x_{n}$ pieces of $n$ units length each, equals

$$
p\left(Y_{i}\right)=\frac{\left(x_{1}+x_{2}+\cdots+x_{n}\right) !\left(\prod_{i=1}^{n} x_{i} !\right)}{A_{01}{ }^{((s+1) n+k)}} \quad\left(Y_{i}=\left(y_{1}, \cdots, y_{n}\right)\right) .
$$

In the partition vectors $Y_{i}$ the index ranges from 1 to $p_{n}(s n+k)$ (number of partitions of $s n+k$ with restrictions $n$ ). Since for $F_{n}(x)$ with $k_{i}=1, d=1, u=1$, formula (2.12) takes the form

$$
w=\lim _{v \rightarrow \infty}\left(A_{01}^{(v-1)} / A_{01}^{(v)}\right)
$$

$$
\left(0<w<1 ; \quad w^{n}+w^{n-1}+\cdots+w-1=0\right) .
$$

Of course, $w$ is a unit of the field $F(w)$ generated by $w$ over the field of rationals. For more extensive investigations of units of algebraic fields see Bernstein and Hasse [6]. Let again $x_{1}, x_{2}, \ldots, x_{n}$ be the roots of $P_{n}(x)$ as in (1.2). Then, for a suitable choice of a convergence radius, we can expand the function $x^{n-1} / P_{n}(x)$ into a convergent power series, viz.

$$
-x^{n-1} /\left(x^{n}+x^{n-1}+\cdots+x-1\right)=\sum_{m=1}^{\infty} a_{m}^{(n)} x^{m-1} .
$$

As has been proved by the author in [2]

$$
a_{m}^{(n)}=F_{m}^{(n)} \quad(m=1,2, \cdots ; n=2,3, \cdots),
$$

so that formula (2.17) takes the form

$$
x^{n-1} /\left(1-x-x^{2}-\cdots-x^{n}\right)=\sum_{m=1}^{\infty} F_{m}^{(n)} x^{m-1} .
$$


Now

$$
\begin{aligned}
1-x-x^{2} & -\cdots-x^{n} \\
& =-P_{n}(x)=-\left(x-x_{1}\right)\left(x-x_{2}\right) \cdots\left(x-x_{n}\right) \\
& =(-1)^{n-1} x_{1} x_{2} \cdots x_{n}\left(1-\frac{x}{x_{1}}\right)\left(1-\frac{x}{x_{2}}\right) \cdots\left(1-\frac{x}{x_{n}}\right) .
\end{aligned}
$$

From $P_{n}(x)=\left(x-x_{1}\right)\left(x-x_{2}\right) \cdots\left(x-x_{n}\right)$ we thus obtain

$$
x^{n}+x^{n-1}+\cdots+x-1=x^{n}-S_{1} x^{n-1}+\cdots+(-1)^{n-1} S_{n-1} x+(-1)^{n} S_{n}
$$

where $S_{i}(i=1, \cdots, n)$ are, as usually, the elementary symmetric functions in the $n$ variables $x_{1}, x_{2}, \cdots, x_{n}$. Since $(-1)^{n} S_{n}=-1$, we obtain $x_{1}, x_{2} \cdots x_{n}=(-1)^{n-1}$ and, therefore,

$$
1-x-\cdots-x^{n}=\left(1-x / x_{1}\right)\left(1-x / x_{2}\right) \cdots\left(1-x / x_{n}\right),
$$

so that formula (2.19) takes the form

$$
x^{n-1} /\left(\prod_{i=1}^{n}\left(1-\frac{x}{x_{i}}\right)\right)=\sum_{m=1}^{\infty} F_{m}^{(n)} x^{m-1},
$$

and since $F_{1}^{(n)}=F_{2}^{(n)}=\cdots=F_{n-1}^{(n)}=0$, we obtain

$$
\begin{gathered}
x^{n-1} /\left(\prod_{i=1}^{n}\left(1-\frac{x}{x_{i}}\right)\right)=\sum_{m=1}^{\infty} F_{n+m-1}^{(n)} x^{n+m-2}, \\
1 /\left(\prod_{i=1}^{n}\left(1-\frac{x}{x_{i}}\right)\right)=\sum_{m=1}^{\infty} F_{n+m-1}^{(n)} x^{m-1}
\end{gathered}
$$

For $|x|<\min \left[\left|x_{i}\right|, i=1, \cdots, n\right]$ we can now expand the above equation into a product of $n$ absolutely convergent power series, viz.

$$
\prod_{i=1}^{n}\left(\sum_{k=0}^{\infty}\left(\frac{x}{x_{i}}\right)^{k}\right)=\sum_{m=1}^{\infty} F_{n+m-1}^{(n)} x^{m-1}
$$

Comparison of coefficients of powers of $x$ on both sides of (2.20) yields

$$
\sum_{j=1}^{n} x_{j}^{-1}=F_{n+1}, \quad \sum_{1 \leq j \leq k \leq n} x_{j}^{-1} x_{k}^{-1}=F_{n+2}
$$

and generally

$$
g_{m}\left(x_{1}, x_{2}, \cdots, x_{n}\right)=F_{n+m}^{(n)}
$$


where $g_{m}\left(x_{1}, x_{2}, \cdots, x_{n}\right)$ is the partition polynomial from (1.3). In view of (2.14) we thus obtain

$$
g_{m}\left(x_{1}, x_{2}, \cdots, x_{n}\right)=A_{01}^{(n+m)} .
$$

3. The explicit formula for $A_{01}^{(v)}$. As was shown in the previous section, it is the calculation of the numbers $A_{01}^{(v)}$ which provide the solution to the problems stated in (i) to (iv). In [3] the author has calculated the values of $A_{01}^{(v)}$ by induction for a few first values of $v$, viz.

$$
\begin{aligned}
& A_{01}^{(n+k)}=2^{k-1}, \quad A_{01}^{(2 n+k)}=2^{n+k-1}-(k+1) 2^{k-2} \\
& A_{01}^{(3 n+k)}=2^{2 n+k-1}-(n+k+1) 2^{n+k-2}+(k-1)(k+2) 2^{k-4} \\
& \quad(k=1, \cdots, n) .
\end{aligned}
$$

On this empirical basis, he then conjectured that the general formula would hold

$$
\begin{array}{r}
A_{01}^{((s+1) n+k)}=2^{k-s-1} \sum_{j=0}^{s}(-1)^{j}\left[\left(\begin{array}{c}
(s-j) n+k \\
j
\end{array}\right)\right. \\
\left.+\left(\begin{array}{c}
(s-j) n+k-1 \\
j-1
\end{array}\right)\right] 2^{(s-j)(n+1)} \\
\quad(s=0,1, \cdots ; k=1, \cdots, n) .
\end{array}
$$

It should be well noted in (3.2) that for $j=s$, the binomial coefficients $\left(\begin{array}{l}\boldsymbol{k} \\ s\end{array}\right)$ and $\left(\begin{array}{c}\boldsymbol{k}-1 \\ s-1\end{array}\right)$ equal zero if $k<s$, or $s<0$. We shall verify formula (3.2) for the cases $s=0,1,2$ to obtain (3.1). For $s=0$ we obtain from (3.2)

$$
A_{01}^{(n+k)}=2^{k-1}\left[\left(\begin{array}{c}
k \\
0
\end{array}\right)+\left(\begin{array}{c}
k-1 \\
-1
\end{array}\right)\right]=2^{k-1}
$$

For $s=1$ we obtain from (3.2)

$$
\begin{aligned}
A_{01}^{(2 n+k)}= & 2^{k-2} \sum_{j=0}^{1}(-1)^{j}\left[\left(\begin{array}{c}
(1-j) n+k \\
j
\end{array}\right)\right. \\
& \left.+\left(\begin{array}{c}
(1-j) n+k-1 \\
j-1
\end{array}\right)\right] 2^{(1-j)(n+1)} \\
& =2^{k-2}\left[2^{n+1}-\left(\left(\begin{array}{c}
k \\
1
\end{array}\right)+\left(\begin{array}{c}
k-1 \\
0
\end{array}\right)\right) 2^{0}\right] \\
& =2^{n+k-1}-(k+1) 2^{k-2} .
\end{aligned}
$$


For $s=2$ we obtain from (3.2)

$$
\begin{aligned}
A_{01}^{(3 n+k)}= & 2^{k-3} \sum_{j=0}^{2}(-1)^{j}\left[\left(\begin{array}{c}
(2-j) n+k \\
j
\end{array}\right)\right. \\
& \left.+\left(\begin{array}{c}
(2-j) n+k-1 \\
j-1
\end{array}\right)\right] 2^{(2-j)(n+1)} \\
= & 2^{k-3}\left[2^{2(n+1)}-\left(\left(\begin{array}{c}
n+k \\
1
\end{array}\right)+\left(\begin{array}{c}
n+k-1 \\
0
\end{array}\right)\right) 2^{n+1}\right. \\
& \left.+\left(\left(\begin{array}{c}
k \\
2
\end{array}\right)+\left(\begin{array}{c}
k-1 \\
1
\end{array}\right)\right) 2^{0}\right] \\
= & 2^{k-3}\left[2^{2(n+1)}-(n+k+1) 2^{n+1}+\frac{1}{2}(k-1)(k+2)\right] \\
= & 2^{2 n+k-1}-(n+k+1) 2^{n+k-2}+(k-1)(k+2) 2^{k-4} .
\end{aligned}
$$

Writing

$$
(s+1) n+k=m+1
$$

in formula (3.2), the latter takes the form

$$
\begin{aligned}
A_{01}^{(m+1)}=\sum_{j=0}^{s}(-1)^{j}\left[\left(\begin{array}{c}
m+1-n-j n \\
j
\end{array}\right)\right. & \\
& \left.+\left(\begin{array}{c}
m-n-j n \\
j-1
\end{array}\right)\right] 2^{m-n-j(n+1)} .
\end{aligned}
$$

It should be emphasized that in (3.4) the values of $m$ are $m=n$, $n+1, \cdots$ Equalizing the binomial coefficients

$$
\left(\begin{array}{c}
m+1-n-j n \\
j
\end{array}\right) \text { or }\left(\begin{array}{c}
m-n-j n \\
j-1
\end{array}\right)
$$

to zero for $m+1-n-j n<j$ or $<0$, formula (3.4) can now be written

$$
\begin{aligned}
A_{01}^{(m+1)}=\sum_{j=0}^{\infty}(-1)^{j}\left[\left(\begin{array}{c}
m+1-n-j n \\
j
\end{array}\right)\right. & \\
& \left.+\left(\begin{array}{c}
m-n-j n \\
j-1
\end{array}\right)\right] 2^{m-n-j(n+1)} .
\end{aligned}
$$

Substituting 


$$
\left(\begin{array}{c}
m-n-j n \\
j-1
\end{array}\right)=\left(\begin{array}{c}
m-n-j n+1 \\
j
\end{array}\right)-\left(\begin{array}{c}
m-n-j n \\
j
\end{array}\right)
$$

in (3.5) we obtain

$$
\begin{aligned}
A_{01}^{(m+1)}=\sum_{j=0}^{\infty}(-1)^{j}\left[2\left(\begin{array}{c}
m+1-n-j n \\
j
\end{array}\right)\right. & \\
& \left.-\left(\begin{array}{c}
m-n-j n \\
j
\end{array}\right)\right] 2^{m-n-j(n+1)},
\end{aligned}
$$

or, finally,

$$
\begin{aligned}
A_{01}^{(m+1)}= & \left(\sum_{j=0}^{\infty}(-1)^{j}\left(\begin{array}{c}
m+1-n-j n \\
j
\end{array}\right) 2^{m-n+1-j(n+1)}\right) \\
& -\left(\sum_{j=0}^{\infty}(-1)^{j}\left(\begin{array}{c}
m-n-j n \\
j
\end{array}\right) 2^{m-n-j(n+1)}\right) .
\end{aligned}
$$

Presume now that formula (3.5) or (3.6) are correct for $m=t, t+1$, $\cdots, t+n-1$. By definition of $A_{01}^{(0)}$ we obtain

$$
A_{01}^{(t+n+1)}=\sum_{j=1}^{n} A_{01}^{(t+j)},
$$

so that, in virtue of (3.6)

$$
\begin{gathered}
A_{01}^{(n+t+1)=} \sum_{i=1}^{n}\left(\sum_{j=0}(-1)^{j}\left(\begin{array}{c}
t+i-n-j n \\
j
\end{array}\right) 2^{t+i-n-j(n+1)}\right. \\
\left.-\sum_{j=0}(-1)^{j}\left(\begin{array}{c}
t+i-1-n-j n \\
j
\end{array}\right) 2^{t+i-1-n-j(n+1)}\right) \\
=\sum_{j=0}(-1)^{j} \sum_{i=1}^{n}\left(a_{i}-a_{i-1}\right)=\sum_{j=0}(-1)^{j}\left(a_{n}-a_{0}\right),
\end{gathered}
$$

where

$$
a_{i}=\left(\begin{array}{c}
t+i-n-j n \\
j
\end{array}\right) 2^{t+i-n-j(n+1)}
$$

thus 


$$
\begin{aligned}
& A_{01}^{(n+t+1)} \\
& =\sum_{j=0}(-1)^{j}\left[\left(\begin{array}{c}
t-j n \\
j
\end{array}\right) 2^{t-j(n+1)}-\left(\begin{array}{c}
t-n-j n \\
j
\end{array}\right) 2^{t-n-j(n+1)}\right] \\
& =\sum_{j=0}(-1)^{j}\left(\begin{array}{c}
t-j n \\
j
\end{array}\right) 2^{t-j(n+1)} \\
& +\sum_{j=0}(-1)^{j+1}\left(\begin{array}{c}
t-n-j n \\
j
\end{array}\right) 2^{t-n-j(n+1)} \\
& =\sum_{j=0}(-1)^{j}\left(\begin{array}{c}
t-j n \\
j
\end{array}\right) 2^{t-j(n+1)} \\
& +\sum_{j=1}(-1)^{j}\left(\begin{array}{c}
t-j n \\
j-1
\end{array}\right) 2^{t+1-j(n+1)} \\
& =\sum_{j=0}(-1)^{j}\left(\begin{array}{c}
t-j n \\
j-1
\end{array}\right) 2^{t+1-j(n+1)} \\
& +\sum_{j=0}(-1)^{j}\left(\begin{array}{c}
t-j n \\
j
\end{array}\right) 2^{t-j(n+1)} \\
& =\sum_{j=0}(-1)^{j}\left(\begin{array}{c}
t-j n \\
j-1
\end{array}\right) 2^{t-j(n+1)}+\sum_{j=0}(-1)^{j}\left(\begin{array}{c}
t-j n \\
j-1
\end{array}\right) 2^{t-j(n+1)} \\
& +\sum_{j=0}(-1)^{j}\left(\begin{array}{c}
t-j n \\
j
\end{array}\right) 2^{t-j(n+1)} \\
& =\sum_{j=0}(-1)^{j}\left(\begin{array}{c}
t-j n+1 \\
j
\end{array}\right) 2^{t-j(n+1)}+\sum_{j=0}(-1)^{j}\left(\begin{array}{c}
t-j n \\
j-1
\end{array}\right) 2^{t-j(n+1)} \\
& =\sum_{j=0}(-1)^{j}\left[\left(\begin{array}{c}
t-j n+1 \\
j
\end{array}\right)+\left(\begin{array}{c}
t-j n \\
j-1
\end{array}\right)\right] 2^{t-j(n+1)} \\
& =\sum_{j=0}(-1)^{j}\left[\left(\begin{array}{c}
t+n+1-n-j n \\
j
\end{array}\right)+\left(\begin{array}{c}
t+n-n-j n \\
j-1
\end{array}\right)\right] 2^{t+n-n-j(n+1)},
\end{aligned}
$$

which indeed is formula (3.5) for $m=t+n$, since (3.5) was correct for $m=s n+k, \quad s n+k+1, \cdots, \quad s n+k+n-1=(s+1) n+k-1, \quad(k=1$, $\cdots, n ; s=1,2)$.

For practical purposes we shall use, instead of formula (3.5) formula (3.2). We now calculate easily 


$$
\begin{aligned}
A_{01}^{(4 n+k)=} & A_{01}^{((3+1) n+k)} \\
= & 2^{k-4} \sum_{j=0}^{3}(-1)^{j}\left[\left(\begin{array}{c}
(3-j) n+k \\
j
\end{array}\right)\right. \\
& \left.+\left(\begin{array}{c}
(3-j) n+k-1 \\
j-1
\end{array}\right)\right] 2^{(3-j)(n+1)} \\
= & 2^{k-4}\left[2^{3(n+1)}-\left(\left(\begin{array}{c}
2 n+k \\
1
\end{array}\right)+\left(\begin{array}{c}
2 n+k-1 \\
0
\end{array}\right)\right) 2^{2(n+1)}\right. \\
& +\left(\left(\begin{array}{c}
n+k \\
2
\end{array}\right)+\left(\begin{array}{c}
n+k-1 \\
1
\end{array}\right)\right) 2^{n+1} \\
\left.-\left(\left(\begin{array}{c}
k \\
3
\end{array}\right)+\left(\begin{array}{c}
k-1 \\
2
\end{array}\right)\right) 2^{0}\right] & \\
= & 2^{3 n+k-1}-(2 n+k+1) 2^{2 n+k-2} \\
& +(n+k-1)(n+k+2) 2^{n+k-4} \\
& -\frac{1}{3}(k-2)(k-1)(k+3) 2^{k-5} .
\end{aligned}
$$

The reader will verify easily that this formula also holds, as it should, for $k=1,2,3,4,5$, in spite of negative powers.

4. Explicit solution of problems (i)-(iv). We are now able, by means of formula (3.2), to state the solutions to problems (i)-(iv) as follows:

(i) Substituting in (3.2) $s=t, k=n$, and then $k=1, s=t+1$, and using formula (2.12) with $u=1, v=(t+2) n+1$, we obtain

THEOREM 1. The (only) positive real root of $P_{n}(x)=-1+\sum_{i=1}^{n} x^{i}$ is given by the formula

$$
\begin{aligned}
& w= \lim _{t \rightarrow+\infty} \\
& \cdot \frac{\sum_{j=0}^{t}(-1)^{j}\left[\left(\begin{array}{c}
(t+1-j) n \\
j
\end{array}\right)+\left(\begin{array}{c}
(t+1-j) n-1 \\
j-1
\end{array}\right)\right] 2^{(t+1) n-j(n+1)-1}}{\sum_{j=0}^{t+1}(-1)^{j}\left[\left(\begin{array}{c}
(t+1-j) n+1 \\
j
\end{array}\right)+\left(\begin{array}{c}
(t+1-j) n \\
j-1
\end{array}\right)\right] 2^{(t+1) n-j(n+1)}} \cdot
\end{aligned}
$$

(ii) By similar substitutions we obtain from (3.2)

THEOREM 2. The value of the partition polynomial 


$$
g_{s n+k}\left(x_{1}, x_{2}, \cdots, x_{n}\right)=\sum x_{1}^{m_{1}} x_{2}^{m_{2}} \cdots x_{n}^{m_{n}},
$$

$\sum$ extended over all $m_{i} \geqq 0(i=1, \cdots, n)$ such that

$$
\begin{gathered}
m_{1}+2 m_{2}+\cdots+n m_{n}=s n+k, \quad(s=0,1, \cdots ; k=1, \cdots, n), \\
P_{n}(x)=-1+\sum_{i=1}^{n} x^{i} ; \quad P_{n}\left(x_{i}\right)=0,
\end{gathered}
$$

is given by the formula

$$
\begin{aligned}
& g_{s n+k} \\
& =2^{k-8-1} \sum_{j=0}^{s}(-1)^{j 2^{(s-j)(n+1)}}\left[\left(\begin{array}{c}
(s-j) n+k \\
j
\end{array}\right)+\left(\begin{array}{c}
(s-j) n+k-1 \\
j-1
\end{array}\right)\right] .
\end{aligned}
$$

(iii) From (3.2) we also obtain the explicit formula for the Generalized Fibonacci Number; such formulas, as is well known, exist for the dimensions 2, 3, 4, but not for higher ones; these are solved by

Theorem 3. The generalized Fibonacci numbers of order $n \geqq 2$, viz.

$$
\begin{aligned}
& F_{1}^{(n)}=F_{2}^{(n)}=\cdots=F_{n-1}^{(n)}=0, \quad F_{n}^{(n)}=1, \\
& F_{m}^{(n)}=\sum_{j=0}^{n-1} F_{m-n+j}^{(n)} \quad(m=n, n+1, \cdots),
\end{aligned}
$$

are given explicitly by the formula

$$
F_{(s+1) n+k}^{(n)}=2^{k-s-1}
$$

$$
\cdot \sum_{j=0}^{s}(-1)^{j} 2^{(s-j)(n+1)}\left[\left(\begin{array}{c}
(s-j) n+k \\
j
\end{array}\right)+\left(\begin{array}{c}
(s-j) n+k-1 \\
j-1
\end{array}\right)\right] .
$$

(iv) The solution to the probabilistic problem as stated in the introduction is given by

THEOREM 4. The probability that a fragile stick of $s n+k$ units length $(s=0,1, \cdots ; k=1, \cdots, n)$ breakable into pieces, each consisting of an integral number of units not exceeding $n$, would break into $y_{1}$ pieces of one unit length each, into $y_{2}$ pieces of two units length each, $\cdots$, into $y_{n}$ pieces of $n$ units length each, so that

$$
y_{1}+2 y_{2}+\cdots+n y_{n}=s n+k, \quad Y_{i}=\left(y_{1}, y_{2}, \cdots, y_{n}\right)
$$

is given by the formula 


$$
=\frac{p\left(Y_{i}\right)}{2^{k-s-1} \sum_{j=0}^{s}(-1)^{j}\left[\left(\begin{array}{c}
(s-j) n+k \\
j
\end{array}\right)+\left(\begin{array}{c}
(s-j) n+k-1 \\
j-1
\end{array}\right)\right] 2^{(s-j)(n+1)}} .
$$

1. Leon Bernstein, The modified algorithm of Jacobi-Perron, Mem. Amer. Math. Soc. No. 67 (1966). MR 34 \#5274.

2. - The linear Diophantine equation in $n$ variables and its application to generalized Fibonacci numbers, Fibonacci Quart. 6 (1968), 1-63. MR 37 \#1301.

3. - A probability function for partitions, Amer. Math. Monthly 75 (1968), 882-886. MR 38 \#794.

4. C. G. J. Jacobi, Allgemeine Theorie der kettenbruchaehnlichen Algorithmen, in welchen jede Zahl aus drei vorhergehenden gebildet wird, J. Reine Angew. Math. 69 (1868).

5. Oscar Perron, Grundlagen fuer eine Theorie des Jacobischen Kettenbruchalgorithmus, Math. Ann. 64 (1907).

6. Leon Bernstein and Helmut Hasse, a) Einheittenberechnung mittels des JacobiPerronschen Algorithmus, J. Reine Angew. Math. 218 (1965), 51-69. MR 31 \#465. b) An explicit formula for the units of an algebraic number field of degree $n \geqq 2$, Pacific J. Math. 30 (1969), 293-365.

Illinois Institute of Technology, Chicago, Illinois 60616 